\title{
FunFam protein families improve residue level molecular function prediction
}

\author{
Linus Scheibenreif $^{1^{*+}}$ (D), Maria Littmann ${ }^{1{ }^{*} \dagger}$, Christine Orengo ${ }^{2}$ and Burkhard Rost ${ }^{1,3,4,5}$
}

\begin{abstract}
Background: The CATH database provides a hierarchical classification of protein domain structures including a sub-classification of superfamilies into functional families (FunFams). We analyzed the similarity of binding site annotations in these FunFams and incorporated FunFams into the prediction of protein binding residues.

Results: FunFam members agreed, on average, in $36.9 \pm 0.6 \%$ of their binding residue annotations. This constituted a 6.7-fold increase over randomly grouped proteins and a 1.2-fold increase (1.1-fold on the same dataset) over proteins with the same enzymatic function (identical Enzyme Commission, EC, number). Mapping de novo binding residue prediction methods (BindPredict-CCS, BindPredict-CC) onto FunFam resulted in consensus predictions for those residues that were aligned and predicted alike (binding/non-binding) within a FunFam. This simple consensus increased the F1-score (for binding) 1.5-fold over the original prediction method. Variation of the threshold for how many proteins in the consensus prediction had to agree provided a convenient control of accuracy/precision and coverage/recall, e.g. reaching a precision as high as $60.8 \pm 0.4 \%$ for a stringent threshold.

Conclusions: The FunFams outperformed even the carefully curated EC numbers in terms of agreement of binding site residues. Additionally, we assume that our proof-of-principle through the prediction of protein binding residues will be relevant for many other solutions profiting from FunFams to infer functional information at the residue level.
\end{abstract}

Keywords: Protein function, Protein families, Functional families, Binding residue prediction, Protein binding sites, CATH

\section{Background}

Knowledge about the function of proteins is crucial for a wide array of biomedical applications. Public resources such as the Gene Ontology (GO) [1] or the Enzyme Commission (EC) classification system [2] provide hierarchical classifications of protein function (frequently also referred to as gene function). The CATH database classifies all proteins for which the three-dimensional structure (3D) is experimentally known in a hierarchy [3]. CATH has also introduced the concept of superfamilies linking proteins with similar 3D structures and very different sequences [4]. The largest known superfamilies are so large that the two hundred largest cover some

\footnotetext{
* Correspondence: scheibenreif@rostlab.org; http://www.rostlab.org; littmann@rostlab.org; assistant@rostlab.org

†Linus Scheibenreif and Maria Littmann contributed equally to this work. Linus Scheibenreif and Maria Littmann share first authorship.

'Department of Informatics, Bioinformatics \& Computational Biology - i12, TUM (Technical University of Munich), Boltzmannstr. 3, 85748 Garching/ Munich, Germany

Full list of author information is available at the end of the article
}

region in $62 \%$ of known proteins [5]. Given the enormity of the span of these superfamilies, only some members of the same superfamily will function alike. Capturing those that do requires a sub-classification into functional families (called FunFams) [3]. CATH FunFams sub-classifies relatives according to similarity in their predicted specificity determining residues. COPS [6] and SCOP [7] provide two alternatives for classifying proteins according to their 3D structure, and SUPERFAMILY joins the sequence-based and the structure-based view of linking families. These classifications capture mostly taxonomical rather than functional relations [8] while using FunFams allows the prediction of protein functions as assessed by CAFA [9].

One problem in assessing functional protein classifications is the following common logical circularity: classifications are based on function annotations for full-length proteins (rather than functional units such as domains) and the reliability of these classifications are measured applying the same type of annotations

(c) The Author(s). 2019 Open Access This article is distributed under the terms of the Creative Commons Attribution 4.0 International License (http://creativecommons.org/licenses/by/4.0/), which permits unrestricted use, distribution, and 
[10]. In the assessment of machine-learning, developers spend substantially resources to evade such a circularity through careful cross-validation and, nevertheless, fail all too often [11-13]. Since cross-validation is much less common for database annotations [14], the circularity is even more difficult to avoid. Here, we side-stepped such a vicious circle by using the similarity of binding residues between proteins as a proxy for functional similarity. Functionally similar proteins are expected to share binding residues that facilitate their common functional task, making it possible to infer similarity in overall function from similarity in binding sites. Since the annotation of protein function, e.g. through GO or EC numbers, often precedes the experimental unravelling of molecular details, our molecular proxy effectively removed the circularity thereby providing an independent means of assessing functional classifications. We added another element, namely results from two methods predicting binding residues exclusively through information available from the sequence (dubbed BindPredict-CCS and BindPredict$C C$ [15]). The development of the method neither used GO nor EC numbers, nor CATH nor FunFams, instead the most important signal originated from evolutionary couplings [16]. We hypothesized that if FunFams extracted relevant information about function, we would find this in the consistency of predicted binding residues within FunFams (more explicitly binding residues would agree more within than between FunFams). If true, we expected to be able to leverage the FunFams clustering to filter binding residue predictions as exemplified by two methods tested (Fig. 1).

\section{Results}

Binding residues agree for FunFams, less so for EC

After omitting all proteins without binding residue annotations (not in the PDB), those with conflicting sequence or annotation lengths, those with duplicate entries (each UniProt identifier once in each FunFam), and families with single members, 7,172 sequences from 1,856 FunFams were left. The average binding residue similarity score for these 1,856 FunFams was $36.9 \pm 0.6 \%$ (Table 1); on average each family had $3.9 \pm 0.1$ proteins (Additional file 1: Figure S1). The average similarity score for randomly constructed sequence families was $5.5 \pm 0.2 \%$. Thus, the binding residue similarity within the same FunFam was 6.7 -fold higher than that between "random families".

To put the FunFam results into perspective of other resources, we analyzed three popular resources in the same way, namely PROSITE [20, 21], Pfam [22], and EC classes [2]. Four thousand ninety sequences in our FunFam dataset mapped to 588 different PROSITE patterns. The average binding residue similarity for these groups was $25.7 \pm 0.8 \%$ (compared to $29.5 \pm 0.8 \%$ similarity within FunFams computed on the same dataset). Three thousand five hundred thirty sequences in our FunFam

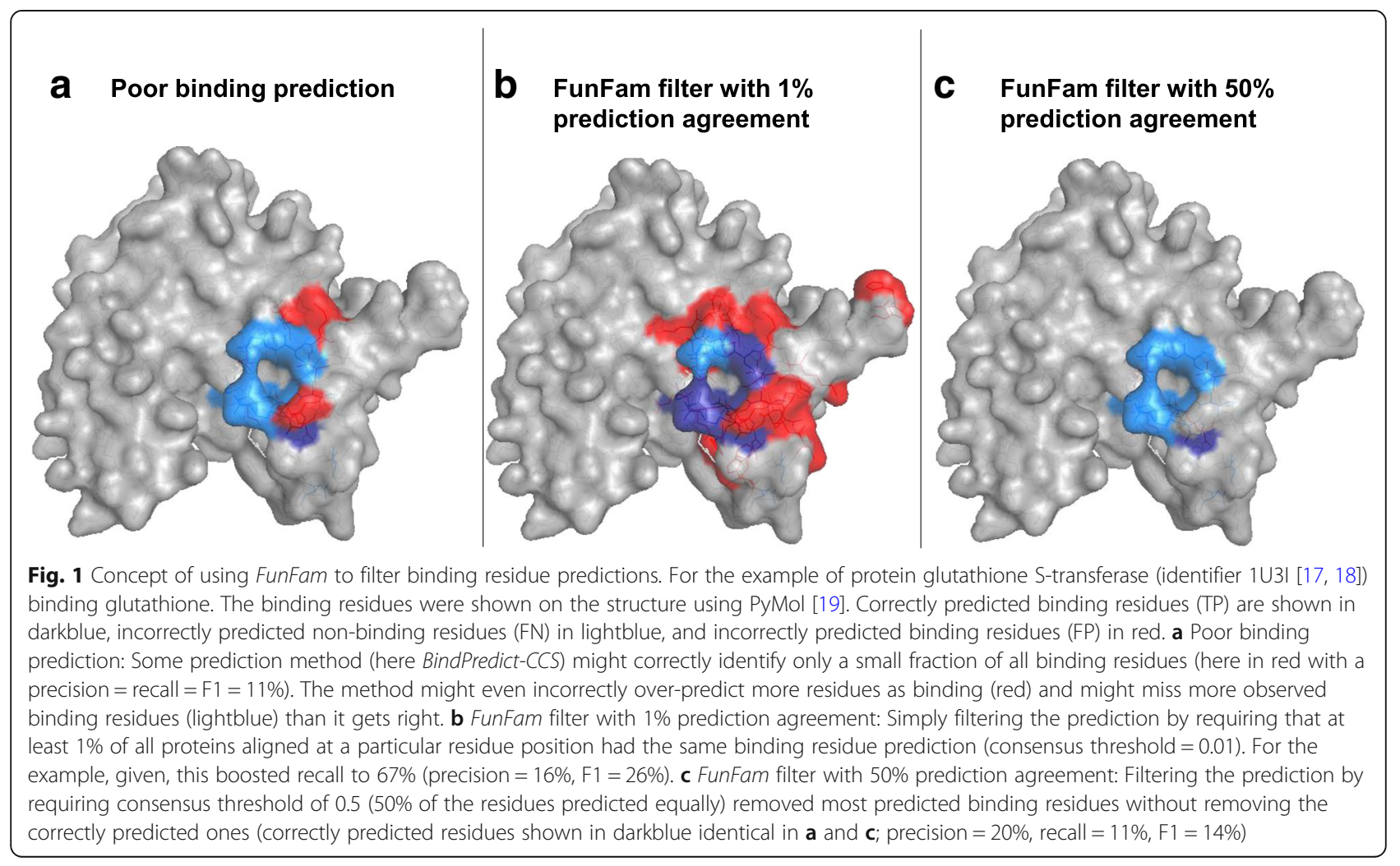


Table 1 Average binding residue similarity for FunFams and EC-numbers ${ }^{\text {a }}$

\begin{tabular}{llll}
\hline Group & Number of families & Number of proteins & $\begin{array}{c}\text { Average binding residue } \\
\text { similarity (Eq. 1) }\end{array}$ \\
\hline Same FunFams & 1856 & 7172 & $36.9 \pm 0.6$ \\
Same EC numbers & 1080 & 5789 & $29.9 \pm 0.8$ \\
Same FunFams, EC-FunFams subset & 1103 & 4143 & $38.6 \pm 0.8$ \\
Same EC numbers, EC-FunFams subset & 833 & 4143 & $34.5 \pm 0.9$ \\
Same EC, different FunFam & 771 & 2893 & $9.6 \pm 0.4$ \\
Same FunFam, different EC & 404 & 2817 & $27.0 \pm 1.0$ \\
Same EC, same superfamily & 1006 & 4445 & $38.0 \pm .0 .01$ \\
Same EC, different superfamily & 435 & 1155 & $5.22 \pm 0.01$
\end{tabular}

${ }^{a}$ Same FunFams: proteins within same FunFam; Same EC-numbers: proteins with identical EC number; EC-FunFams subset: same subset used for both similarity calculation with FunFams and within EC classes; Same EC different FunFam: subset of proteins with identical EC number classified into different FunFams; Same FunFam different EC: subset of proteins from same FunFam with different EC numbers; Same EC, same superfamily: proteins with identical EC number grouped into a structural superfamily; Same EC, different superfamily: proteins with identical EC number grouped into different superfamilies; \pm : refers to one standard error

dataset mapped 656 Pfam families which had an average binding residue similarity of $26.2 \pm 0.3 \%$ (compared to $30.6 \pm 0.8 \%$ similarity within FunFams computed on the same dataset). Both approaches outperformed randomly grouped sequences more than five-fold but performed worse than FunFams (1.2-fold).

For comparison with a specialized functional classification, we also computed binding residue similarity for the EC numbers classification. Our FunFam dataset contained 5,789 proteins with 1,080 different EC numbers (all had complete annotations for all four levels of the EC number; the remaining 1,383 proteins were ignored for this investigation). The average binding residue similarity for proteins with the same four-level EC number was $29.9 \pm 0.8 \%$ (Table 1), a 5.4-fold increase over random. The binding residue similarity was higher for FunFams than for EC numbers across all similarity levels (Fig. 2). The average for FunFams was 1.2-fold higher (1.1-fold on same dataset) than for EC numbers. The same was true for particular points in the distribution, e.g. for families with $100 \%$ binding residue similarity (Fig. 2: rightmost values), and those with, e.g. $60 \%$ or $50 \%$ similarity (Fig. 2 :

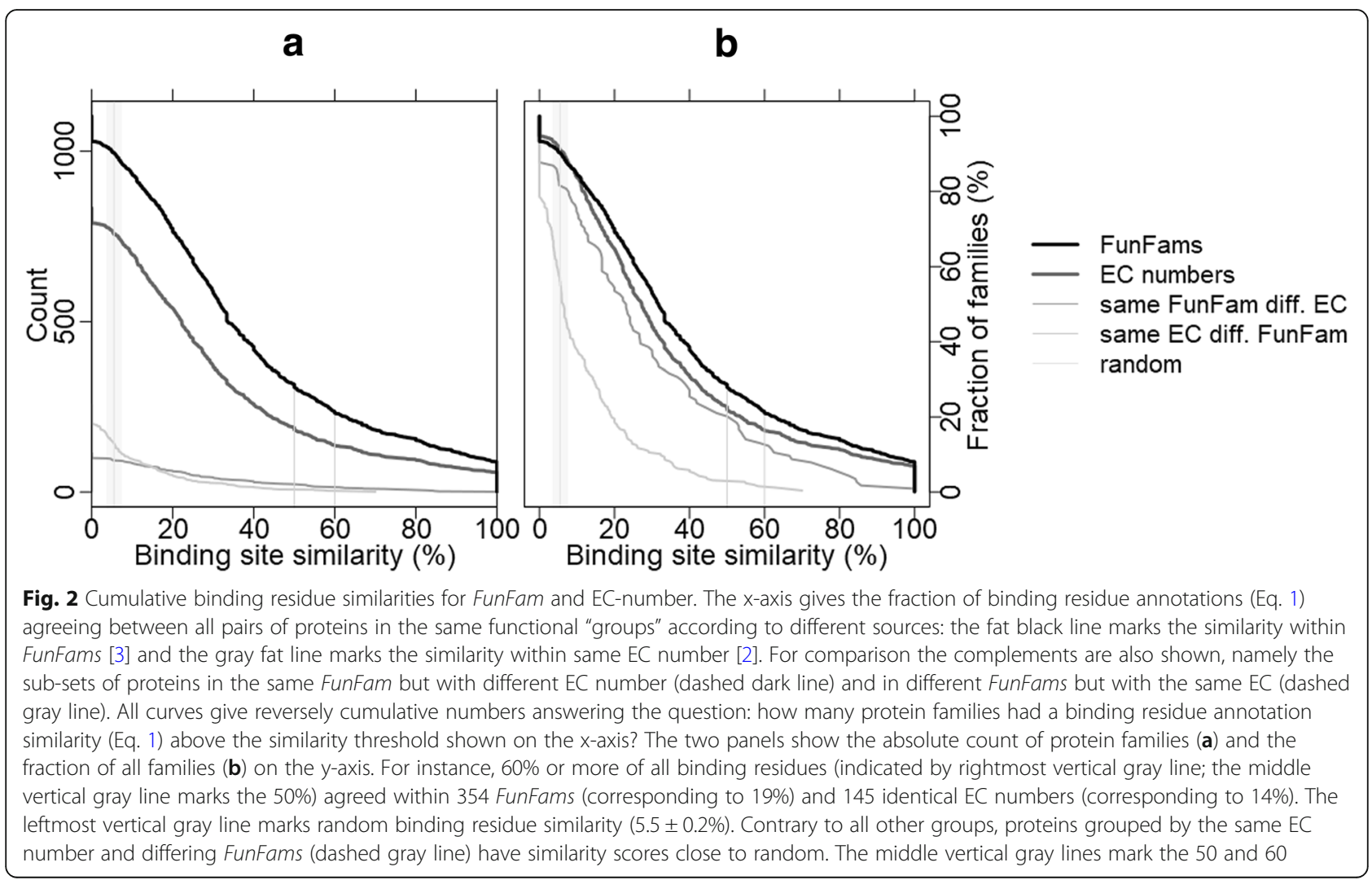


light gray vertical lines on right and in middle). Conversely, the fraction of those with binding residue similarity levels close to random (Fig. 2: intersection of lines with gray shading on left) were higher for EC than for FunFams, except at zero, i.e. no binding residue similarity (FunFams 6.95\% vs. EC numbers 6.67\%).

To use the largest subsets possible, we calculated the similarity within FunFams and within EC classes on different subsets. To ensure that performance differences did not largely result from differences in the sub-sets, we re-computed all values for a smaller subset identical to both $(4,143$ proteins grouped into 1,103 FunFams and into $833 \mathrm{EC}$ classes). On this subset, the average binding residue similarity for proteins within the same FunFam was $38.6 \pm 0.8 \%$ that within the same EC class was $34.5 \pm 0.9 \%$, i.e. FunFam performed 1.1-times better than EC numbers supporting the statistically more relevant results for the larger subsets (Table 1).

We also extracted all proteins with identical EC number classified into different FunFams (if more than one found, one representative selected randomly). This resulted in 771 groups (each representing one EC number) with 2,893 proteins. These groups had an average binding residue similarity of $9.6 \pm 0.4 \%$ (Table 1, Fig. 2: gray dashed line). Conversely, we computed the average similarity for proteins in the same FunFam but with different EC numbers (if several sequences in a FunFam had the same EC, we picked one at random). This yielded 404 groups (each representing one FunFam) with 2,817 proteins; the average binding residue similarity in this group was $26.8 \pm 0.1 \%$ (Table 1, Fig. 2: dark dashed line). Along a similar line, we found that EC number annotations became more consistent when constrained by the superfamily. The average binding residue similarity for identical EC numbers rose to $38.0 \pm 0.01 \%$ (1.2fold improvement) for the subset of proteins with the same EC number and the same superfamily (with 4,445 proteins from 1006 EC numbers: Table 1). Notably, 69\% of all EC numbers that occurred in a superfamily grouped into its most frequent FunFam. Furthermore, we found that the binding residue similarity of protein pairs with the same EC number but grouped into two different superfamilies dropped to a random level of $5.22 \pm 0.01$ (Table 1). The dataset contained 1,155 such proteins from $435 \mathrm{EC}$ numbers.

\section{Binding annotation transfer within FunFams raises precision}

Homology-based inference implies the following transfer: if proteins $\mathrm{P} 1$ and $\mathrm{P} 2$ are sufficiently sequence similar (e.g. $\operatorname{PIDE}(\mathrm{P} 1, \mathrm{P} 2)<\mathrm{T})$, experimental annotations obtained for P1 could be transferred to P2. We applied such a homology-based inference by transferring binding residue annotations from one member of a FunFam to all other members. This resulted in an F1 score of $37.97 \pm 0.01 \%$ (Precision $=49.03 \pm 0.01 \%$, Recall $=47.52 \pm 0.01 \%)$ and an MCC of $0.36 \pm 0.0002$. This was further evidence for the high degree of functional similarity within FunFams.

\section{Binding residue prediction improved through FunFam filter} The methods BindPredict-CCS and BindPredict-CC predict binding residues through cumulative coupling scores and clustering coefficients derived from DI scores [15]. We applied these methods to 470 proteins from 138 FunFams. For that set, the prediction with cumulative coupling scores reached an F1-score of $10.5 \pm 1 \%$ and the prediction with clustering coefficients an F1 $=14.2 \pm 1 \%$. Building consensus predictions at consensus thresholds of 0.01 from all predictions for members of a FunFam raised the F1-score for cumulative coupling scores to $16.2 \pm 0.8 \%$ corresponding to a 1.5-fold increase (Additional file 1: Figure S2). At the same threshold, the corresponding values for precision, recall, and accuracy were $18.3 \pm 0.1 \%$ (Eq. 2), $29.8 \pm 0.2 \%$ (Eq. 3) and $71.1 \pm 0.1 \%$ (Eq. 4) respectively (Fig. 3a showing precision and recall). This corresponded to roughly 1.4-fold increase for precision, one-third decrease for recall and a one-tenth decrease for accuracy (data not shown). For predictions based on clustering coefficients, the F1-score increased 1.3fold to $18.4 \pm 1 \%$ (Additional file 1: Figure S2). Precision decreased 0.7 -fold to $17.5 \pm 1 \%$ (Eq. 2) while recall reached $49.5 \pm 1 \%$ (Eq. 3), a 2.0-fold improvement (Fig. 3b). The accuracy was $55 \pm 1 \%$ (1.3-fold decrease). The MCC was very low for all predictions. Nevertheless, the consensus prediction still increased the MCC about two-fold (2.1fold for BindPredict-CCS at consensus threshold 0.01; 2.0-fold at 0.1; Additional file 1: Figure S3).

Varying the consensus threshold at which a binding prediction was included into the consensus, i.e. the number of proteins within a FunFam for which the same residue had to be predicted as binding, provided a convenient way for tuning precision and recall. At a consensus threshold of 1.0, precision reached $60.8 \pm 0.4 \%$ (2.5-fold increase over standard method) for the cumulative couplings method (Fig. 3a) and $44.0 \pm 0.4 \%$ (1.9-fold increase over standard method) for clustering coefficient-based predictions (Fig. 3b). At this conservation threshold, about three residues were, on average, predicted in each protein as binding and at least one residue was predicted for $55.2 \%$ of the proteins. For comparison: for the clustering coefficients, 10.4 residues were predicted as binding per protein and at least one residue was predicted for $34.4 \%$ of the proteins.

\section{Consensus prediction vs. machine learning prediction from bindPredictML17}

To compare the consensus predictions with the results of a more sophisticated binding residue prediction method not using information from FunFams, we 
a

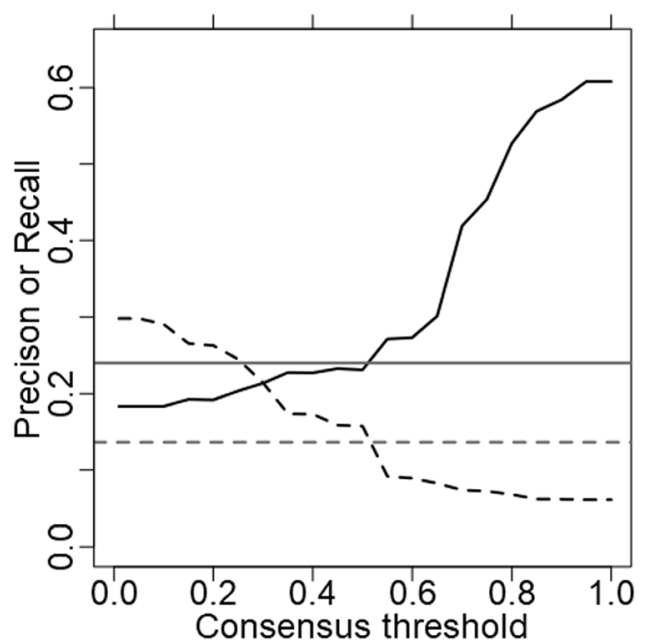

b

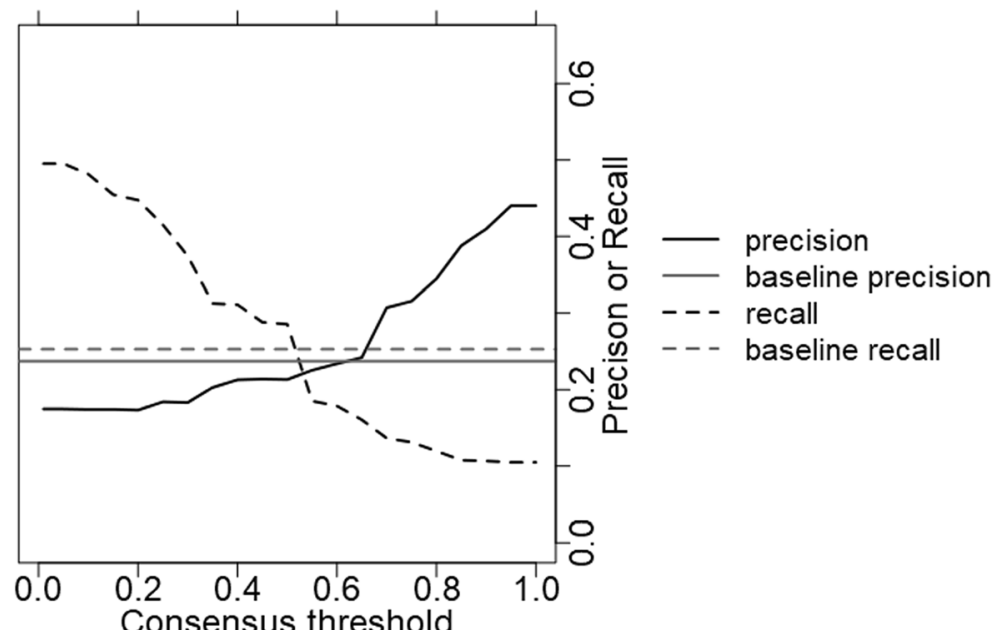

Fig. 3 Leveraging FunFams to better predict binding residues. The horizontal lines indicate the performance estimates for precision (Eq. 2) and recall (Eq. 3) of BindPredict-CCS and BindPredict-CC baseline predictions not using FunFams. Predictions are refined by constructing consensus predictions for the FunFams. The x-axes give different thresholds in terms of what fraction of the FunFams members need to have a binding prediction for a particular residue in order to label that residue as binding in the consensus prediction: from at least one (0.01) to all (1.0). Depending on the threshold, both precision and recall significantly increase over the standard prediction method. The two panels illustrate the improvement over two slightly different baseline prediction methods: a BindPredict-CCS using the cumulative couplings-based input features. In this case precision increases up to $61 \pm 4 \%$. Panel $\mathbf{b}$ shows the performance improvement for BindPredict-CC using the clustering coefficient-based input features. For low thresholds, these predictions reach recall up to $50 \pm 2 \%$

applied bindPredictML17 [23] on 114 sequences from the FunFam dataset that were also part of the development set of bindPredictML17. For these proteins, bindPredictML17 reached $\mathrm{F} 1=25.85 \pm 0.01 \%$ (precision $=$ $31.40 \pm 0.02 \%$, recall $=32.59 \pm 0.02 \%)$. Applying the FunFam filter at a consensus threshold of 0.01 led to $\mathrm{F} 1=$ $14.8 \%$ for BindPredict-CC and F1 $=19.0 \%$ for BindPredict-CCS. The highest recall of $43.6 \%$ was reached for BindPredict-CC at a consensus threshold of 0.01 , and the highest precision of $50.7 \%$ for BindPredict-CCS for a threshold of 1.0.

\section{Discussion}

The significantly higher binding residue similarity within the same FunFams than within "random families" strongly supported our hypotheses that protein binding residues proxy protein function, and that FunFams succeed in the classification of residue binding sites. However, the average agreement between known binding sites remained below $40 \%$. This might be explained by five challenges. Firstly, there might be problems with FunFams. Secondly, too many binding sites might remain unknown. Thirdly, some experimentally annotated binding residues might not be based on cognate ligands. Fourthly, binding sites might shift without substantially affecting function. Fifthly, function might differ more between related proteins than expected. Although only the first of those five possible explanations fell within the scope of this work, we might speculate about an upper limit for the amount of the problem that could be contributed by the other four explanations. To address it, we investigated how well-known residue binding sites would agree for the popular automated resources PROSITE [20, 21] and Pfam [22], as well as, for the expertcurated EC numbers classification, considered to be the most precise existing manual curation of protein function for the subset of enzymes.

Protein families as described by PROSITE patterns or Pfam families have a clearly higher similarity in binding residue annotations than randomly grouped sequences showing that they succeed in correctly classifying proteins into families. However, the average binding residue similarity was even higher within the same FunFam.

On sequences grouped by their EC number, the substantial increase in binding residue similarity over random demonstrated the fine-grained classification according to catalytic function by the EC number system. Although FunFam classifies proteins automatically rather than driven by expert annotations, the average binding residue similarity was higher within the same FunFam than for identical EC numbers. Keeping the same EC number and removing the constraint "same FunFam" dropped binding residue similarity by about 20 percentage points (Table 1), while FunFams were much more robust against the removal of subsets from the same EC number (half the drop by ten percentage points, Table 1). 
This suggested proteins to have the same EC number only when they originated from the same FunFam. The binding site similarity of EC numbers constrained by $\mathrm{CATH}$ superfamily had a level similar to FunFams, but this did not imply that FunFams "only" add the superfamily classification to EC numbers as revealed by the immense drop for EC without FunFams (Fig. 2: dark dashed line). Hence, FunFams appeared to provide a more fine-grained and more consistent classification of protein function than even such a carefully expert-driven system as the EC numbers.

The FunFam filter managed to halve the difference in F1-score between simple prediction methods relying on only one feature and a state-of-the-art machine-learning approach. Depending on the consensus thresholds, recall or precision reached or even exceeded this approach. However, only the machine-learning approach stroke a good balance between recall and precision, therefore still outperforming the consensus prediction. This suggested that combining the consensus prediction with a more sophisticated binding residue prediction method might yield further improvements from the FunFams filter. We expect this expectation to be limited by the accuracy of the prediction method. In this analysis, we have focused exclusively on predictions available for all proteins of know sequences even for those that do not map to FunFams. Methods based on 3D structure are known to perform much better predictions, and only those can actually aspire to predict binding sites rather than binding residues [24-28].

Binding residue predictions were significantly improved using our FunFam filter. Besides an increase in F1-score, high consensus thresholds yielded high precision. This suggested that all proteins within a FunFam share some binding residues. These might be the key residues most important to maintain function and they can be identified by applying this consensus prediction (Fig. 1c). Lowering the consensus threshold increased recall. This might help to identify unknown binding residues that might be experimentally annotated in only a few FunFam members but might remain to be discovered in others (Fig. 1b). Overall, the fact that predictions agreed within FunFams constituted another, independent way to shine light on the degree to which the sub-classification of CATH superfamilies through FunFam succeeded in automatically classifying functional families. FunFams clearly encode functional information in the form of shared binding residues. This information was, indeed, so consistent that, e.g. binding residue predictions were improved by tapping directly into this information.

In our analysis, we focused on a few measures for the agreement of binding residue annotations and the gain in prediction performance in order to reduce the complexity of the results. As additional measures, we also applied
MCC (Matthew correlation coefficient), Accuracy (or Q2, i.e. percentage of residues correctly predicted/identically annotated in either of the two states binding/non-binding), along with values for different thresholds. None of those measures changed our findings in any relevant relative way (values nominally changed but not in terms of their relative differences). We did not assess scores summarizing the entire distribution of measures such as the ROC curve or the AUC, because we can only calculate those for the baseline predictions from BindPredict-CCS and BindPredict-CC but not for the consensus prediction. The consensus prediction only provides binary labels (binding/non-binding) and lacks probabilities or prediction scores needed to compute a ROC curve. Furthermore, ROC curves using the consensus cut-off as threshold are not meaningful since this cut-off does not control the prediction outcome directly and a maximal cut-off of 1.0 does not necessarily yield false/true positive rates of 1 .

The highest level of improvement in prediction performance through FunFams was about 0.6 (Fig. 3a: top right level of dark line marking precision). We might speculate that this suggested an upper limit for the problem of all the above five challenges (missing annotations, noncognate ligands, shifts in binding sites neutral with respect to function and changes of function between related proteins): at most those issues matter for $40 \%$ of all binding residues, i.e. the glass is already more than half full.

\section{Conclusions}

FunFams derived from CATH super-families aim at grouping functionally related proteins. Here, we showed that known binding residues are over six times (6.7) more consistent (Eq. 1) between sequences of the same FunFam than between sequences of different FunFams. FunFams automatically classify all proteins, nevertheless, they covered binding residue similarity about $20 \%$ better (1.2-fold increase, 1.1-fold on same dataset) than the expert curated EC numbers (Enzyme Classification) identical on all four digits for the particular classification of enzymes and about 20\% (1.2-fold increase) better than PROSITE patterns or Pfam families. On top, the high similarity of binding residues for proteins with the same EC number mostly originated from the same FunFam. These results clearly demonstrated FunFams to capture functional information at the residue level with a degree of detail not matched by the EC numbers. This information was so helpful that it directly improved binding residue prediction based on evolutionary couplings (BindPredict-CCS, BindPredict-CC). A simple consensus prediction over many FunFam members yielded a substantially (30-50\%) improved F1 score of $0.184 \pm 0.009$. Tuning the consensus threshold adjusted the precision/recall: for the highest possible threshold (1.0, meaning all members of the FunFam have to have that particular residue predicted as 
binding) precision reached as high as $60 \%(60.8 \pm 0.4 \%)$. At this threshold, at least one binding residue was predicted for every other protein (55\%). The major power of this simple analysis might lie in its generality: there was no reason why not any prediction method will improve by implementing the same filtering step.

\section{Methods}

\section{Data set}

The FunFam dataset is available online through the CATH database $[3,29]$. Protein domain sequences from the same CATH superfamily are sub-classified into different FunFams by first performing profile-profile based comparisons between clusters of closely related sequences and applying an iterative, agglomerative clustering protocol to progressively merge clusters having profile-profile scores above a threshold. This creates a tree of putative functional relationships between clusters which is then cut by identifying differences in conserved specificity determining residues, and other likely 'functional determinants', between clusters. FunFams have been demonstrated to be much more structurally and functionally coherent than CATH superfamilies [3]. The FunFam dataset used here consisted of 1,267,077 protein domain sequences from 14,928 FunFams. Since FunFams are based on domains, there can be multiple FunFam assignments for the same protein. Binding site annotations were available for 7,172 proteins from 1,856 FunFams.

\section{Binding residue annotations}

Binding residue annotations for sequences were obtained from the Protein Data Bank (PDB; information taken from SITE records including the description "binding site") [17]. PDB structures were mapped to UniProt sequences through SIFT [30]. Note: we only used labels for individual binding residues without attempting to group this $1 \mathrm{D}$ information into 3D binding sites.

\section{PROSITE}

PROSITE $[20,21]$ is a database of biologically meaningful patterns. These patterns are derived from multiple sequence alignments (MSAs) of related sequences even when the relationship is too distant to be identified solely by pairwise sequence comparisons. PROSITE patterns typically span 10-20 residues that are assumed to be important for the function of all proteins containing this pattern [21].

\section{Pfam families}

Pfam [22] is a hidden Markov model profile base database of protein families. It provides multiple sequence alignments of protein sequences and classifies entries into the types family, domain, motif, repeat, coiled coil or disordered. Pfam strives for high quality and completeness using a highly automated procedure [31].

\section{EC numbers}

EC numbers classify enzymes through a four-level hierarchy [2]. For example, enzymes classified as EC: 1.1.1.are oxidoreductases (first level), acting on the $\mathrm{CH}-\mathrm{OH}$ group of electron donors (second level), with NAD+ or $\mathrm{NADP}+$ as an electron acceptor (third level). EC numbers might constitute the most reliable annotation of protein function despite some limits [32].

\section{Binding residue similarity}

Binding residue annotations were compared between proteins through a simple similarity measure (Eq. 1), namely the sum over all binding residues annotated between two aligned sequences normalized by the maximum number of binding residues in one of the two.

$$
\begin{gathered}
\text { pairwise_similarity }\left(X^{n}, Y^{n}\right)=\frac{100}{n} \sum_{i=1}^{n} z_{i} \text { with } z_{i} \\
=\left\{\begin{array}{c}
1, \text { if } x_{i} \in\left(y_{1}, \ldots, y_{m}\right) \\
0, \text { otherwise }
\end{array} \text { and } \mathrm{n} \geq \mathrm{m}\right. \text { w.l.o.g. }
\end{gathered}
$$

$\mathrm{X}$ and $\mathrm{Y}$ are vectors containing the indices of binding residues mapped to an alignment of the corresponding sequences. This measure was generalized to comparisons of $\mathrm{M}$ proteins $(M>2)$ by averaging over all $M^{*}(M-1) / 2$ pairwise similarities.

\section{Random binding residue similarity}

The random similarity score was constructed as the average similarity score of randomly chosen sequences grouped into "random families". Size and number of the "random families" was chosen to mimic the structure of the FunFam dataset. ClustalW aligned these randomly selected sequences [33] providing the MSA to compute the random binding residue similarity.

\section{Homology-based inference within one FunFam}

To assess the similarity of binding residue annotations within FunFams we adopted a simple approach toward homology-based inference: The binding residue annotation of one FunFam member P1 was transferred to all other members and evaluated in comparison to the original annotation of P1. This was done in an iterative procedure such that ultimately the annotation was transferred and evaluated for each member for which it was available.

\section{Binding residue prediction}

In this work, we focus on two basic methods derived from bindPredictML17 [23]: BindPredict-CCS and BindPredict-CC, which are based on cumulative coupling scores (CCS) and clustering coefficients (CC) computed from evolutionary couplings $[15,23]$. The evolutionary couplings were obtained by applying three publicly 
available tools, namely EVcouplings [34] using jackhmmer [35] to build families and Freecontact [36] to infer DI (Direct Information) scores through mean-field direct coupling analysis (more details published elsewhere $[15,23])$ from these MSAs.

\section{Consensus prediction}

The consensus prediction for a FunFam was built by combining the predicted labels (binding/non-binding) of residues from all sequences in the FunFam such that there was a prediction for each column in the MSA. A column in the MSA was predicted as binding if the fraction of sequences for which that residue was predicted as binding exceeded a consensus threshold. The consensus can be chosen variable to optimize precision, recall, or F1 score depending on the application. The higher the threshold, the fewer residues were predicted as binding. For instance, a consensus threshold of 0.3 for a particular residue implied that $30 \%$ of all proteins aligning at that residue position (i.e. those without insertions or deletions at that position) predicted this residue as binding.

\section{Performance measures}

For simplicity, we used only the following standard measures to measure the success of the consensus prediction. With the standard labels TP (true positives: correctly predicted binding residues), TN (true negatives: correctly predicted as non-binding), FP (false positives: predicted as binding not observed experimentally; note that many of these constitute missing annotations, i.e. will turn into TP with greater experimental coverage), and FN (false negatives: predicted as non-binding, observed experimentally to bind). We used:

$$
\begin{aligned}
& \text { Precision }=100 \cdot \frac{T P}{T P+F P} \\
& \text { Recall }=100 \cdot \frac{T P}{T P+F N} \\
& \text { Accuracy }=100 \cdot \frac{T P+T N}{T P+T N+F N+F P} \\
& F 1=2 \cdot \frac{\text { Precision } * \text { Recall }}{\text { Precision }+ \text { Recall }} \\
& M C C=100 \cdot \frac{T P \cdot T N-F P \cdot F N}{\sqrt{(T P+F P)(T P+F N)(T N+F P)+(T N+F N)}}
\end{aligned}
$$

All results were stated with their corresponding standard error. The standard error was calculated as standard deviation divided by the square root of $n-1 . n$ is the number of proteins and the standard deviation is obtained from the distribution of performances per protein.

\section{Additional file}

Additional file 1: Supporting Online Material containing additional figures. (DOCX $5332 \mathrm{~kb}$ )

\begin{abstract}
Abbreviations
1D: One-dimensional - information representable in a string such as "binding residues"; 3D structure: Three-dimensional coordinates of protein structure; CATH: Class Architecture Topology Homology; CC: Clustering coefficients; CCS: Cumulative coupling scores; EC: Enzyme Commission (number capturing the hierarchy of enzyme function); FunFam: Functional protein family; GO: Gene Ontology (functional annotation of proteins); MSA: Multiple sequence alignment; PDB: Protein Data Bank
\end{abstract}

\section{Acknowledgements}

Thanks to Tim Karl (TUM), for invaluable help with hardware and software; to Inga Weise (TUM) for support with many other aspects of this work and thanks to Thomas Hopf (TUM, Harvard, OmicScouts) and all the other developers of EVcouplings (Harvard University) for making their program publicly available. Particular thanks to Sayoni Das (UCL) for building FunFams and for help with using this resource. Thanks also to the anonymous reviewers who helped substantially to improve the paper. Last, not least, thanks to Helen Berman (PDB Rutgers), loannis Xenarios (Swiss-Prot, SIB, Geneva), Francisco Melo (PDIdb, Santiago) and their crews for maintaining excellent databases and to all experimentalists who enabled this analysis by making their data publicly available.

\section{Authors' contributions}

LS analyzed and interpreted the FunFam dataset, performed consensus predictions, and was the main person responsible for writing the manuscript. ML developed BindPredict-CC and BindPredict-CCS, guided analysis of the data, and was a major contributor to manuscript writing. CO provided the FunFam data, assisted with questions regarding FunFams, provided important comments to refine the analysis, and contributed to manuscript writing. BR supervised the work over the entire time and proofread the manuscript. All authors read and approved the final manuscript.

\section{Funding}

This work was supported by the German Research Foundation (DFG) and the Technical University of Munich (TUM) in the framework of the Open Access Publishing Program and the Bavarian Ministry for Education through funding to the TUM paying for the positions of the authors. The funding body did not play any role in the design of the study and collection, analysis, and interpretation of data and in writing the manuscript.

\section{Availability of data and materials}

The datasets generated and analyzed during the current study are available in the GitHub repository https://github.com/Rostlab/FunFamsConsensus. The FunFam dataset is available through the CATH database, http://cathdb.info.

Ethics approval and consent to participate

Not applicable.

\section{Consent for publication}

Not applicable.

\section{Competing interests}

The authors declare that they have no competing interests.

\section{Author details}

${ }^{1}$ Department of Informatics, Bioinformatics \& Computational Biology - i12, TUM (Technical University of Munich), Boltzmannstr. 3, 85748 Garching/ Munich, Germany. ${ }^{2}$ Department of Structural and Molecular Biology, University College London, London WC1E 6BT, UK. ${ }^{3}$ Institute for Advanced Study (TUM-IAS), Lichtenbergstr. 2a, 85748 Garching/Munich, Germany. ${ }^{4}$ TUM School of Life Sciences Weihenstephan (WZW), Alte Akademie 8, Freising, Germany. ${ }^{5}$ Department of Biochemistry and Molecular Biophysics \& New York Consortium on Membrane Protein Structure (NYCOMPS), Columbia University, 701 West, 168th Street, New York, NY 10032, USA. 
Received: 18 April 2019 Accepted: 9 July 2019

Published online: 18 July 2019

\section{References}

1. Ashburner M, Ball CA, Blake JA, Botstein D, Butler H, Cherry JM, Davis AP, Dolinski K, Dwight SS, Eppig JT. Gene ontology: tool for the unification of biology. Nat Genet. 2000;25(1):25.

2. Bairoch A. The ENZYME database in 2000. Nucleic Acids Res. 2000;28(1):304-5.

3. Sillitoe I, Cuff AL, Dessailly BH, Dawson NL, Furnham N, Lee D, Lees JG, Lewis TE, Studer RA, Rentzsch R. New functional families (FunFams) in CATH to improve the mapping of conserved functional sites to 3D structures. Nucleic Acids Res. 2012;41(D1):D490-8.

4. Orengo CA, Michie AD, Jones S, Jones DT, Swindells MB, Thornton JM. CATH - a hierarchic classification of protein domain structures. Structure. 1997:5:1093-108.

5. Dessailly BH, Nair R, Jaroszewski L, Fajardo JE, Kouranov A, Lee D, Fiser A Godzik A, Rost B, Orengo C. PSI-2: structural genomics to cover protein domain family space. Structure. 2009;17(6):869-81.

6. Suhrer SJ, Wiederstein M, Gruber M, Sippl MJ. COPS - a novel workbench for explorations in fold space. Nucleic Acids Res. 2009;37(Web Server issue): W539-44.

7. Murzin AG, Brenner SE, Hubbard T, Chothia C. SCOP: a structural classification of proteins database for the investigation of sequences and structures. J Mol Biol. 1995;247(4):536-40.

8. Pethica RB, Levitt M, Gough J. Evolutionarily consistent families in SCOP: sequence, structure and function. BMC Struct Biol. 2012;12(1):27.

9. Zhou N, Jiang Y, Bergquist TR, Lee AJ, Kacsoh BZ, Crocker AW, Lewis KA, Georghiou G, Nguyen HN, Hamid MN, et al. The CAFA challenge reports improved protein function prediction and new functional annotations for hundreds of genes through experimental screens. bioRxiv. 2019:653105. https://doi.org/10.1101/653105.

10. Sillitoe I, Cuff AL, Dessailly BH, Dawson NL, Furnham N, Lee D, Lees JG, Lewis TE, Studer RA, Rentzsch R, et al. New functional families (FunFams) in CATH to improve the mapping of conserved functional sites to $3 \mathrm{D}$ structures. Nucleic Acids Res. 2013;41(Database issue):D490-8.

11. Moult J, Fidelis K, Kryshtafovych A, Rost B, Tramontano A. Critical assessment of methods of protein structure prediction-round VIII. Proteins. 2009;77(S9):1-4.

12. Rost $B$, Eyrich $V$. EVA: large-scale analysis of secondary structure prediction. Proteins Struct Funct Genet. 2001;45(Suppl 5):S192-9.

13. Hamp T, Rost B. More challenges for machine-learning protein interactions. Bioinformatics. 2015;31(10):1521-25.

14. Mitchell AL, Attwood TK, Babbitt PC, Blum M, Bork P, Bridge A, Brown SD, Chang HY, El-Gebali S, Fraser MI, et al. InterPro in 2019: improving coverage, classification and access to protein sequence annotations. Nucleic Acids Res. 2018;47(D1):D351-60.

15. Schelling M. Predicting protein binding sites through machine learning with evolutionary couplings. Master's thesis. Munich: Technical University of Munich, Ludwig-Maximilians-Universität München; 2017.

16. Hopf TA, Colwell LJ, Sheridan R, Rost B, Sander C, Marks DS. Three-dimensional structures of membrane proteins from genomic sequencing. Cell. 2012;149(7): 1607-21.

17. Berman HM, Westbrook J, Feng Z, Gilliland G, Bhat TN, Weissig H, Shindyalov IN, Bourne PE. The Protein Data Bank. Nucleic Acids Res. 2000:28:235-42.

18. Chomilier J, Vaney M-C, Labesse G, Trottein F, Capron A, Mormon J-P. The crystal structure of gluthatione S-transferase from Schistosoma mansoni. https://www.rcsb.org/pages/policies\#References.

19. Schrodinger L. The PyMOL Molecular Graphics System, Version 1.8; 2015.

20. Sigrist CJ, de Castro E, Cerutti L, Cuche BA, Hulo N, Bridge A, Bougueleret L, Xenarios I. New and continuing developments at PROSITE. Nucleic Acids Res. 2013;41(Database issue):D344-7.

21. Sigrist CJ, Cerutti L, Hulo N, Gattiker A, Falquet L, Pagni M, Bairoch A, Bucher P. PROSITE: a documented database using patterns and profiles as motif descriptors. Brief Bioinform. 2002;3(3):265-74.

22. El-Gebali S, Mistry J, Bateman A, Eddy SR, Luciani A, Potter SC, Qureshi M, Richardson LJ, Salazar GA, Smart A, Sonnhammer ELL, Hirsh L, Paladin L, Piovesan D, Tosatto SCE, Finn RD. The Pfam protein families database in 2019. Nucleic Acids Res. 2019;47(D1):D427-32.

23. Schelling M, Hopf TA, Rost B. Evolutionary couplings and sequence variation effect predict protein binding sites. Proteins. 2018;86(10):1064-74.

24. Aloy P, Russell RB. Understanding and predicting protein assemblies with 3D structures. Comp Funct Genomics. 2003;4(4):410-5.
25. Betts MJ, Wichmann O, Utz M, Andre T, Petsalaki E, Minguez P, Parca L, Roth FP, Gavin AC, Bork P, et al. Systematic identification of phosphorylation-mediated protein interaction switches. PLoS Comput Biol. 2017;13(3):e1005462.

26. Duran-Frigola M, Siragusa L, Ruppin E, Barril X, Cruciani G, Aloy P. Detecting similar binding pockets to enable systems polypharmacology. PLoS Comput Biol. 2017;13(6):e1005522.

27. Lewis TE, Sillitoe I, Andreeva A, Blundell TL, Buchan DW, Chothia C, Cozzetto D, Dana JM, Filippis I, Gough J, et al. Genome3D: exploiting structure to help users understand their sequences. Nucleic Acids Res. 2015;43(Database issue):D382-6.

28. Wass MN, Kelley LA, Sternberg MJ. 3DLigandSite: predicting ligand-binding sites using similar structures. Nucleic Acids Res. 2010;38(Web Server issue): W469-73.

29. Dawson NL, Lewis TE, Das S, Lees JG, Lee D, Ashford P, Orengo CA, Sillitoe I. CATH: an expanded resource to predict protein function through structure and sequence. Nucleic Acids Res. 2017;45(D1):D289-95.

30. Velankar S, Dana JM, Jacobsen J, van Ginkel G, Gane PJ, Luo J, Oldfield TJ, O'donovan C, Martin M-J, Kleywegt G. SIFTS: structure integration with function, taxonomy and sequences resource. Nucleic Acids Res. 2012;41(D1): D483-9.

31. EL Sonnhammer SE, R. Durbin: Pfam: a comprehensive database of protein domain families based on seed alignments. Proteins. 1997;3:405-20.

32. Mahlich $Y$, Steinegger $M$, Rost B, Bromberg Y. HFSP: high speed homology-driven function annotation of proteins. Bioinformatics. 2018; 34(13):i304-12

33. Larkin MA, Blackshields G, Brown N, Chenna R, McGettigan PA, McWilliam H, Valentin F, Wallace IM, Wilm A, Lopez R. Clustal W and Clustal X version 2.0. Bioinformatics. 2007;23(21):2947-8.

34. Hopf TA, Schärfe CP, Rodrigues JP, Green AG, Kohlbacher $O$, Sander C, Bonvin AM, Marks DS. Sequence co-evolution gives 3D contacts and structures of protein complexes. eLife. 2014;3:e03430

35. Finn RD, Clements J, Arndt W, Miller BL, Wheeler TJ, Schreiber F, Bateman A, Eddy SR. HMMER web server: 2015 update. Nucleic Acids Res. 2015;43(W1): W30-8.

36. Kaján L, Hopf TA, Kalaš M, Marks DS, Rost B. FreeContact: fast and free software for protein contact prediction from residue co-evolution. BMC Bioinformatics. 2014;15(1):85

\section{Publisher's Note}

Springer Nature remains neutral with regard to jurisdictional claims in published maps and institutional affiliations.

Ready to submit your research? Choose BMC and benefit from:

- fast, convenient online submission

- thorough peer review by experienced researchers in your field

- rapid publication on acceptance

- support for research data, including large and complex data types

- gold Open Access which fosters wider collaboration and increased citations

- maximum visibility for your research: over $100 \mathrm{M}$ website views per year

At $\mathrm{BMC}$, research is always in progress.

Learn more biomedcentral.com/submission 